\title{
MODELLING THE EFFECTS OF MESOSTRUCTURE ON ELECTRONIC APPLICATIONS OF POLYMER THIN LAYERS
}

\author{
Marta M. D. Ramos*, Helena M. G. Correia \\ Departamento de Física, Universidade do Minho, Campus de Gualtar, \\ 4710-057 Braga, Portugal
}

\begin{abstract}
There is considerable experimental evidence that the microstructure has important consequences for polymer-based electronic and optoelectronic applications, but few theoretical and computational models account for it. We produced several realisations of polymer networks exhibiting specific arrangements of $\mathrm{C}_{4 \mathrm{n}+2} \mathrm{H}_{2 \mathrm{n}+4}$ molecules at the mesoscopic scale and we carried out computer experiments in which bipolar charge carriers were injected in the polymer system from the appropriate electrodes.

Our results show that polymer microstructures resulting from different arrangements of polymer molecules have significant effects on the competition between charge trapping, current transport and recombination within the polymer layer. It was found that current efficiency increases non-linearly with the external applied electric field, the effect being more pronounced for molecular orientations parallel to the electrode surface. In contrast, recombination efficiency shows an opposite behaviour since no significant charge accumulation within the polymer layer is predicted. However, the space-charge effects due to electrons and holes are responsible for most of the recombination events not occurring in neither long or short chains.
\end{abstract}

Keywords: Mesoscopic modelling, mesostructure effects, charge transport and recombination, conducting polymers.

\footnotetext{
* Corresponding Author: Tel:+351 253 604330; Fax:+351 253678 981; E-mail: marta@ fisica.uminho.pt
} 


\section{Introduction}

Over the past decades, there has been considerable effort in advancing thin film polymer devices for electronic and optoelectronic applications [1]. These devices use films with thickness of only few hundred nanometres and different molecular arrangements. Their electrical properties are determined by the collective properties of charge transport hopping between polymer molecules.

In trying to develop high-efficiency polymer based devices in the field of microelectronics, it has become clear that the details of molecular alignment have a large impact on charge transport and, therefore, device efficiencies $[2,3]$. The small size of these devices allows their functioning to be simulated in computers. As a result, mesoscopic modelling techniques $[4,5]$ can be used to replace the costly and lengthy experimental runs needed to study the effects of polymer mesostructure on the electrical properties of those devices.

Device modelling for single-layer polymer light-emitting diodes is the essence of the present work. In the mesoscopic model used for that purpose we defined a three-dimensional arrangement of polydiacetylene (PDA) molecules, taking the form of $\mathrm{C}_{4 \mathrm{n}+2} \mathrm{H}_{2 \mathrm{n}+4}$, between the electrodes (anode and cathode) and rules for bipolar charge injection, transport and recombination. In this paper we study the effect of different molecular alignments relative to the electrode surface on current, trapping and recombination efficiencies for several external applied electric fields, as well as the distribution of recombination events within the polymer thin film.

\section{Model and simulation details}

The polymer films were composed of PDA straight chains, considered as rigid rods, with chain lengths varying from 2 to 8 monomer units which were taken from Gaussian distribution

of lengths of mean value 5. The PDA films with a thickness of $100 \mathrm{~nm}$ and density of 0.15 
$\mathrm{g} / \mathrm{cm}^{3}$ were built between two planar electrodes with an area of $400 \mathrm{~nm}^{2}$ by placing the PDA molecules randomly in the gap between the electrodes with a specific molecular orientation relative to the electrode surface. Three types of molecular alignment were considered in this work: parallel, perpendicular and randomly oriented relative to the electrodes. Furthermore during the construction of the polymer film we applied periodic boundary conditions to the directions parallel to the electrode surface and a minimum inter-molecular distance of $0.5 \mathrm{~nm}$ was considered, based on self-consistent quantum molecular dynamics calculations [6].

When an external electric field is applied across the electrodes, one hole is injected from the anode and one electron from the cathode at each computer iteration (which corresponds to the time in which one inter-chain jump takes place). The holes and electrons migrate through the polymer film under the influence of the local electric field which is the sum of the external applied field, the field of the other charges in transit within the polymer layer and the field due to electrode polarization.

The intra-chain motion takes place before the hopping process between neighbouring chains. It allows the charge to move along the chain from the injection site to the chain-end favoured by the direction of the local electric field if its strength along the chain direction is greater than $1.60 \times 10^{8} \mathrm{~V} / \mathrm{m}$ for holes and $2.35 \times 10^{8} \mathrm{~V} / \mathrm{m}$ for electrons [6], otherwise it moves towards the centre of the chain which is energetically favourable. The motion of charge carriers due to hopping is simulated via a generalized Monte Carlo technique. A detailed description of this technique is given in reference [5] and other references therein. The other molecular properties of PDA needed for the mesoscopic calculations described above were also taken from reference [6]. A dielectric constant of 2.3 [7] was used in the calculation of the local electric field.

When charges of opposite sign meet on the same chain they recombine to form an exciton. According to the experimental results of Sixl [8] only short PDA chains with less than 6 
monomer units can emit light in the visible region of the electromagnetic spectrum due to relaxation from the excited state to the ground state.

\section{Results and discussion}

Depending on processing conditions, semi-conducting conjugated polymer molecules can adopt different orientations relative to the electrode surface which has important consequences for the electrical properties of these materials.

In order to study the relationship between polymer structure and the electric characteristics of polymer based devices, we have calculated the proportions of injected charge (electrons and holes) which are trapped, recombined and which cross the polymer film to carry a current for three different polydiacetylene mesostructures with the molecular axis either parallel, perpendicular or randomly oriented relative to the electrode surface. In the numerical simulations we followed the evolution of injected charges from the initial state to the steady state.

Figure 1 shows the calculated current efficiency as a function of the external applied electric field for the three different polymer alignments considered. It was found that current efficiency increases as the applied electric field increases for all polymer structures, the effect being more pronounced for molecular chains randomly oriented relative to the electrode surface. This is probably due to the random space distribution of the polymer molecules within a film leading to the existence of the larger number of short charge transfer percolating paths at random orientation as compared to the case of the parallel or perpendicular orientation. For the case of molecular rods parallel to the electrode surface, our results are in qualitative agreement with the non-ohmic current-voltage characteristics of PDA thin films produced by spin coating [9].

The electrons and holes accumulation inside the PDA thin film in the steady state as a function of the external applied electric field are shown in figure 2. Only a few percent of the 
injected charge of both signs is accumulated within the polymer layer and most of them are accumulated close to the collected electrode. For each polymer mesostructure, the amount of charge accumulated within the polymer layer does not seem to depend strongly on the strength of applied electric field. The accumulation of charge is more pronounced for the molecular alignment perpendicular to the electrode surface, the effect being stronger for electron accumulation.

Since the amount of accumulated charge within the polymer layer is very low for all polymer mesostructures considered and it does not seem to change significantly with the strength of the applied electric field, recombination efficiency (see figure 3) shows an opposite behaviour to current efficiency.

In the steady state, the distribution of bipolar charge accumulated within the polymer thin film leads to an internal electric field in the region far from the electrodes lower than the electric field close to the electrodes, which favours a random walk of the charges in this region. As a consequence, there is an increase in the number of recombination events in the central region of the polymer film (figure 4). The recombination distribution profile depends on the molecular arrangement within the polymer film. It may also be noted that as the external applied electric field increases, the number of the recombination events decreases all over the polymer film but the recombination distribution profile remains roughly similar for each polymer mesostructure.

Our results also show that only a small fraction of the polymer molecules is responsible for the major part of the recombination events within the polymer layer, regardless of the polymer mesostructure. The recombination distribution profile at a given molecular arrangement is mostly dictated by the number of recombination events that takes place in polymer chains with 5 and 6 repeat monomer units. Despite the fact that the number of chains with 6 monomer units is lower than the number of those with 5 monomer units, the number of recombination events that occur in the longer chains dominates at low applied electric field. This situation 
disappears as the applied electric field increases. As the strength of the applied field increases, the number of recombination events that happen in chains with 5, 6 and 7 units decreases, the effect being more pronounced for chains with 6 monomer units. In contrast, the behaviour observed for chains with 4 monomer units is opposite. The number of recombination events in polymer chains with other lengths is found to be negligible.

\section{Conclusions}

Mesoscopic modelling of bipolar charge evolution in polymer thin films, such as those reported here, enables us to shed light on the correlation between polymer mesostructure and the electrical characteristics of the polymer film. A key point of this model is to consider the complexity of the charge transport process in the polymeric systems where intra-molecular and inter-molecular processes all contribute somewhat to the overall process.

In the steady state, changes in molecular arrangements (morphologies) does not seem to affect charge accumulation within the polymer film although they contribute significantly to the competition between current flow and recombination. While much more work is needed to study the role and control of polymer mesostructure in order to improve luminescence in polymer based devices, our results already provide useful guidelines to move towards this goal: the majority of recombination events seems to take place in polymer chains with 5 and 6 monomers, as opposed to longer or shorter polymer chains. The dominance of recombination events in short chains increases with the applied electric field.

\section{Acknowledgement}

This work is part of the research project POCTI/CTM/41574/2001, approved by the Portuguese Foundation for Science and Technology (FCT) and POCTI and supported by the European Community Fund FEDER. One of us (H.M.G.C.) is also indebted to FCT for financial support under PhD grant n ${ }^{\circ}$. SFRH/BD/11231/2002. 


\section{References}

[1] R.H. Friend, R.W. Gymer, A.B. Holmes, J.H. Burroughes, R.N. Marks, C. Taliani, D.D.C. Bradley, D.A. Dos Santos, J.L. Bredas, M. Logdlund, and W.R. Salaneck, Nature 397 (1999) 121.

[2] Y. Shi, J. Liu, and Y. Yang, Journal of Applied Physics 87 (2000) 4254.

[3] W. Geens, S.E. Shaheen, B. Wessling, C.J. Brabec, J. Poortmans, and N.S. Sariciftci, Organic Electronics 3 (2002) 105.

[4] M.M.D. Ramos, and A.M. Stoneham, Computational Materials Science 17 (2000) 260.

[5] M.M.D. Ramos, H.M.G. Correia, R.M. Ribeiro, and A.M. Stoneham, Synthetic Metals 147 (2004) 281.

[6] A.M. Stoneham, M.M.D. Ramos, A.M. Almeida, H.M.G. Correia, R.M. Ribeiro, H. Ness, and A.J. Fisher, Journal of Physics-Condensed Matter 14 (2002) 9877.

[7] T. Hasegawa, T. Okamoto, M. Haraguchi, M. Fukui, T. Koda, and K. Takeda, Japanese Journal of Applied Physics 37 (1998) 5793.

[8] H. Sixl, in D. Blook (Ed), Polydiacetylene, Nijhoff, Amesterdam, 1984, p. 240.

[9] A.N. Aleshin, S.W. Chu, V.I. Kokub, S.W. Lee, J.Y. Lee, S.H. Lee, D.W. Kim, and Y.W. Park, Current Applied Physics 5 (2005) 85. 


\section{List of figure captions}

Figure 1 - Current efficiency (the proportion of charge carriers crossing the polymer film to reach the opposite electrode), through the PDA network as a function of the applied electric field: electrons (closed marks) and holes (open marks). These results were obtained for the following molecular orientations relative to the electrode surfaces: parallel (square), perpendicular (triangle) and random orientation (circle).

Figure 2 - The dependence of holes (a) and electrons (b) accumulated at steady state within the PDA network as a function of the external applied electric field for the following molecular orientations relative to the electrode surfaces: parallel (square), perpendicular (triangle) and random orientation (circle).

Figure 3 - The proportion of injected charge which recombine within the PDA network as a function of the applied electric field for the following molecular orientation relative to the electrode surfaces: parallel (square), perpendicular (triangle) and random orientation (circle).

Figure 4 - The recombination intensity distribution inside the PDA network, as obtained in the numerical simulation, for three different strengths of the external applied electric field [(a) $E_{\text {ext }}=1 \times 10^{8} \mathrm{~V} / \mathrm{m}$; (b) $E_{\text {ext }}=2 \times 10^{8} \mathrm{~V} / \mathrm{m}$; (c) $\left.E_{\text {ext }}=3 \times 10^{8} \mathrm{~V} / \mathrm{m}\right]$ and the following molecular orientations relative to the electrode surfaces: parallel (square), perpendicular (triangle) and random orientation (circle). 
Figure 1

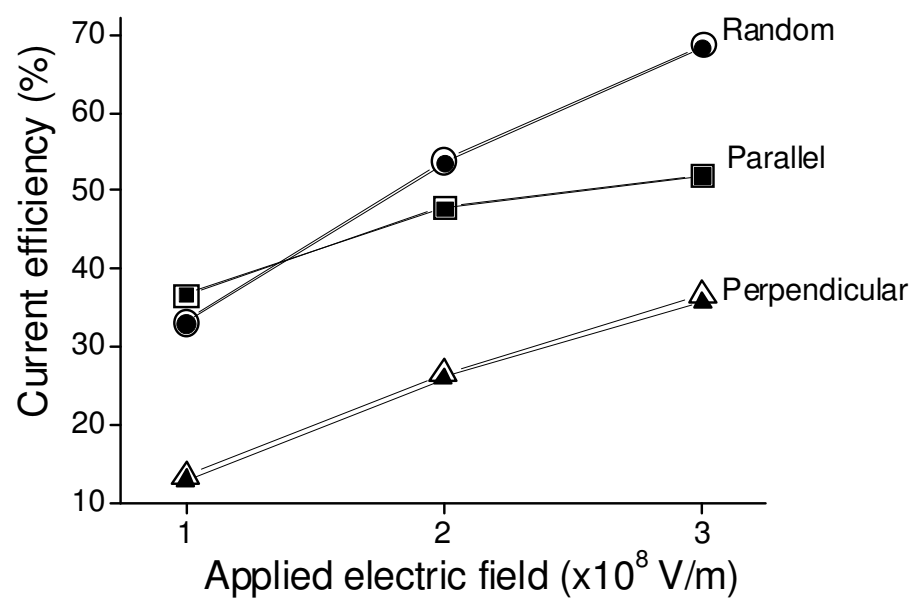


Figure 2
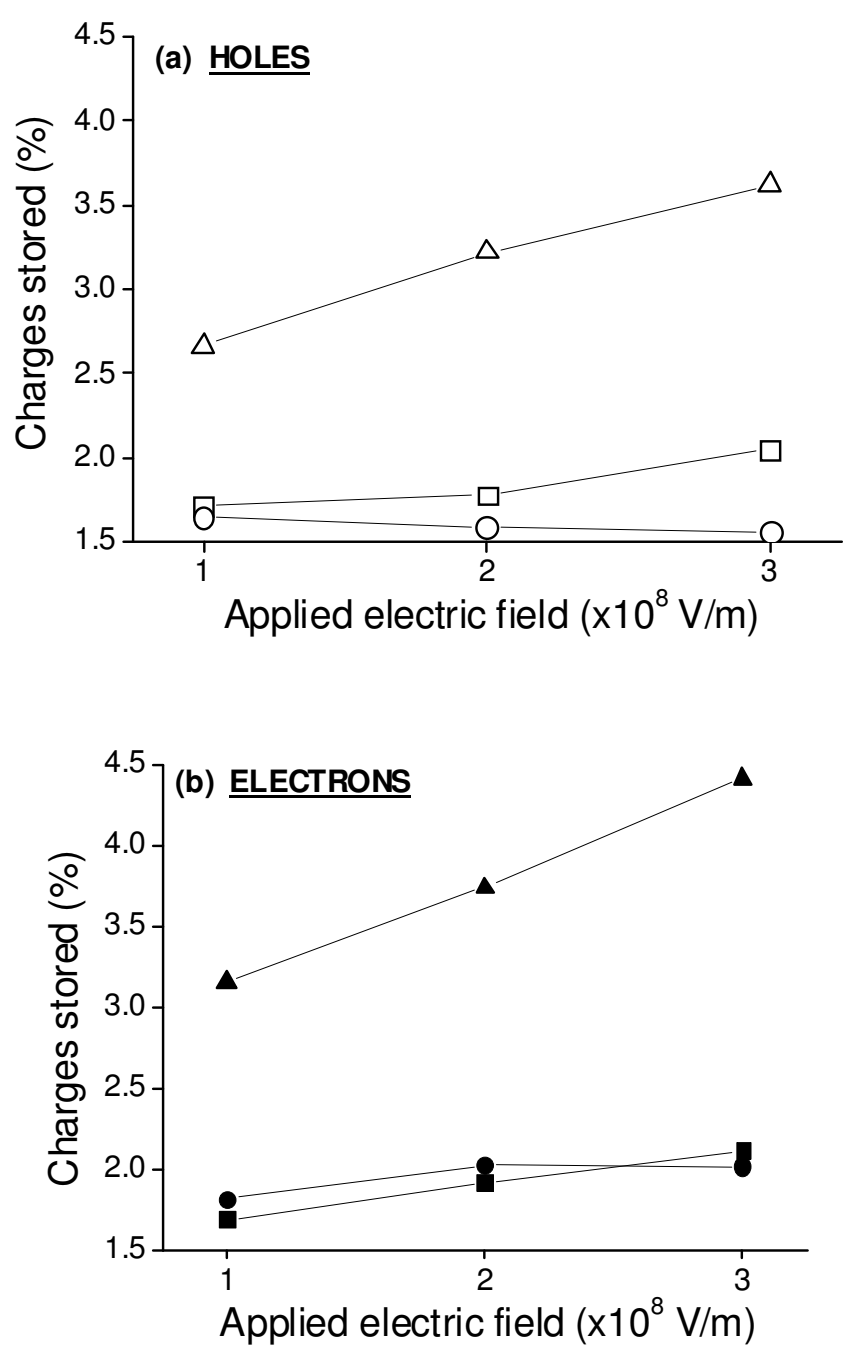
Figure 3

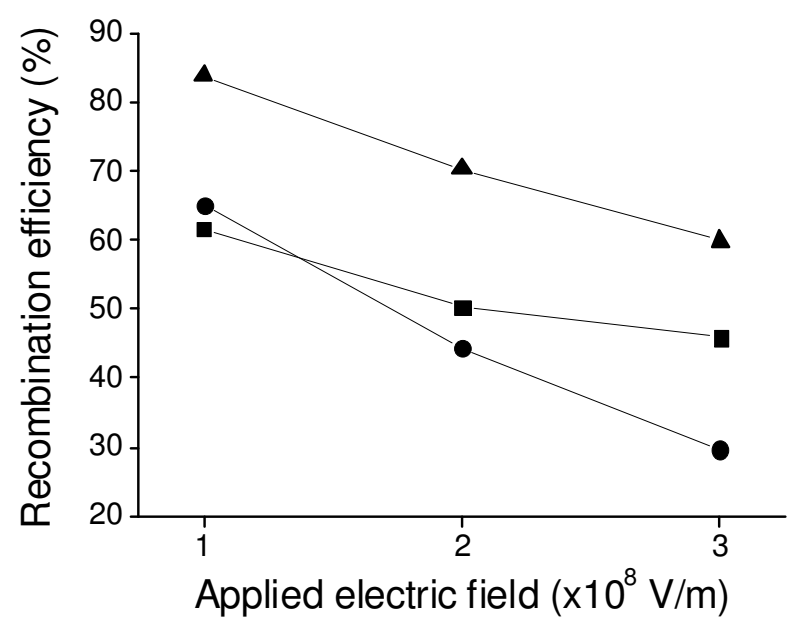


Figure 4
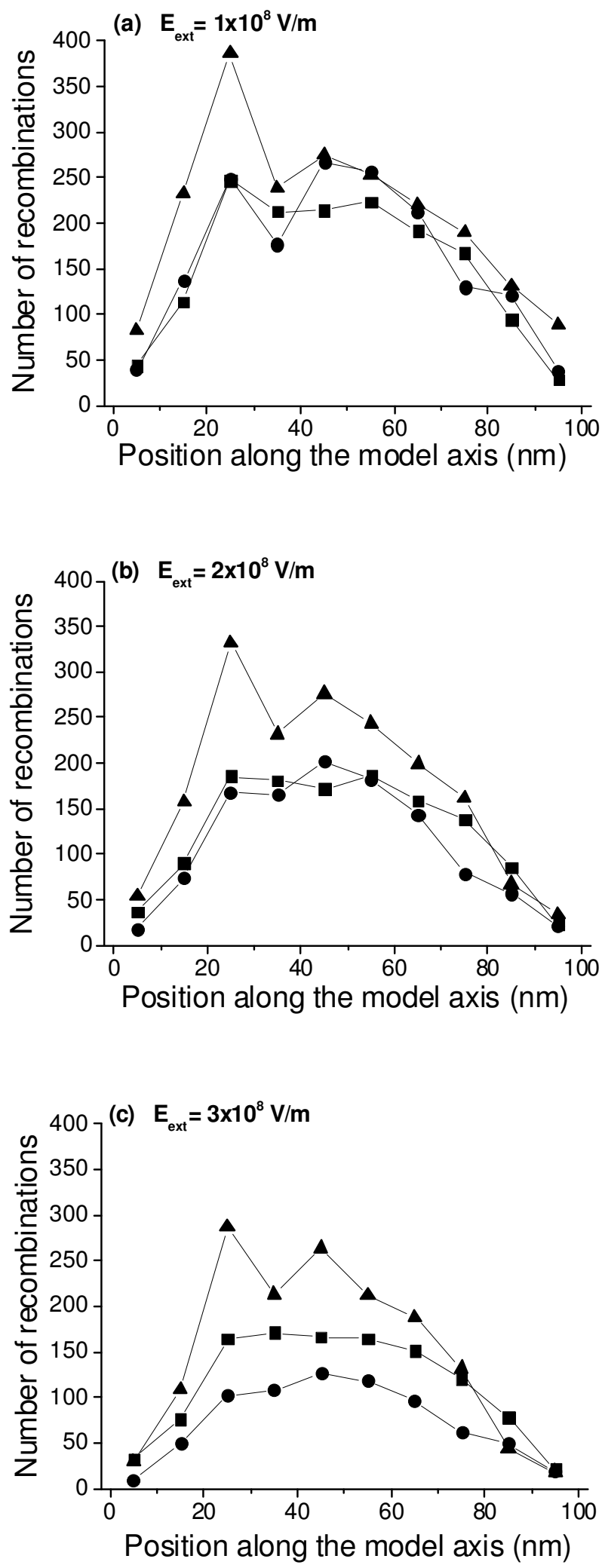